\title{
Modeling and Analysis of Sub-Millimeter-Wave Graphene Switches for On-Wafer Coplanar Transmission Lines
}

\author{
Panagiotis C. Theofanopoulos, Student Member, IEEE, and Georgios C. Trichopoulos, Member, IEEE
}

\begin{abstract}
We present an analysis of graphene loaded transmission line switches. Namely, we propose equivalent circuit models for graphene loaded coplanar waveguides and striplines and examine the switching performance under certain design parameters. As such, the models account for the distributed effects of electrically-large shunt switches in coplanar waveguides and we use the Babinet's principle to derive the respective models for the coplanar stripline transmission lines. Using these models, we identify the optimum design of graphene switches based on transmission line characteristic impedance, scaling factor, graphene shape, and topology (series or shunt). We vary these parameters and obtain the insertion loss and ON/OFF ratio. The extracted results can act as the design roadmap toward an optimum switch topology and emphasize the limitations with respect to fabrication challenges, parasitic effects, and radiation losses. In our models, we use measured graphene values (sheet impedance) instead of theoretical equations, to obtain the actual switching performance. Finally, the proposed equivalent models are crucial for this in-depth study; since, we simulated more than $2,000,000$ configurations, a computationally challenging task with the use of full-wave solvers.
\end{abstract}

Index Terms-Graphene, terahertz, millimeter-waves, switches, modeling, transmission lines

\section{INTRODUCTION}

Millimeter-wave (mmWave) and terahertz (THz) frequencies (100 GHz-3 THz) have been widely exploited for a variety of applications including high-throughput communications, sensing, and imaging, due to their small wavelength and ample bandwidth [1]-[8]. In such applications, reconfiguration, especially through switching, is crucial in enabling radio-frequency (RF) signal processing including carrier modulation/encoding or beam steering in antenna arrays [9]-[15]. At mmWave/THz frequencies, switches can provide many practical solutions without residing to the complexity of more traditional devices, including mixers or phase shifters. For example, a switch can be integrated on an antenna to enable direct signal control and provide 1- or 2-bit quantized phase modulation [9]-[15]. These configurations are mainly categorized as either coded phased arrays (e.g. 1-bit digital phased arrays [9][10]) or reflective surfaces (e.g. reflectarrays [11]-[15]). Such reconfigurable apertures carry out frequency

P.C. Theofanopoulos (ptheofan@asu.edu) and G. C. Trichopoulos (gtrichop@asu.edu) are with the School of Electrical, Computer and Energy Engineering, Arizona State University, Tempe, AZ, USA. independent beamforming, while retaining low DC power consumption and RF losses compared to traditional phased array systems [16]. Moreover, these reconfigurable multielement antenna arrays, which are expected to dominate in at higher frequencies, are less prone to phase quantization errors compared to smaller ones [17]. Switches can typically achieve simpler modulation schemes (e.g. 1-bit phase or amplitude modulation); however, spectral efficiency is not necessarily a requirement at $\mathrm{mmWave} / \mathrm{THz}$ bands, where bandwidth can be orders of magnitude larger than lower RF.

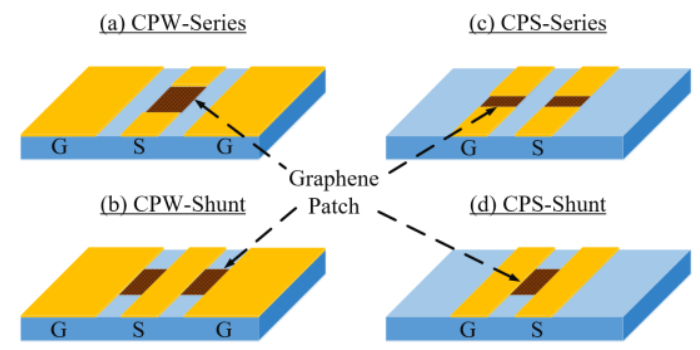

Fig. 1 The proposed CPW and CPS graphene loaded transmission line configurations.

State-of-the-art switches are based on CMOS, SiGe, GaAs, and InP technologies [18]-[28], and provide insertion loss (IL) less than $3 \mathrm{~dB}$ with switching ratios (R) up to $15 \mathrm{~dB}$ at $300 \mathrm{GHz}$. However, the downsides of such switch devices are 1) their limited cutoff frequencies (typically less than $300-500 \mathrm{GHz}$ ) and 2) the fabrication cost increases dramatically when integrated into large arrays with thousands of elements because of the chip real-estate cost [29]. Specifically, the performance of the aforementioned switches is limited by the parasitics and mobility constraints exhibited in $\mathrm{mmWave} / \mathrm{THz}$ frequencies (at room temperatures) [30]. To compensate for these limitations, more advanced technology nodes are required, thus increasing the fabrication cost significantly. For example, mmWave/THz reconfigurable reflectarrays with an aperture of $20 \mathrm{~mm} \times 20$ $\mathrm{mm}$ can host more than 1,600 antenna elements at $300 \mathrm{GHz}(\lambda / 2$ sampling). To implement such an array with state-of-the-art methods requires at least 1,600 switches (one per antenna), which can be manually assembled as chips on the aperture (to reduce the cost) or have an entire wafer of $400 \mathrm{~mm}^{2}$ with all the 
switches integrated. Therefore, scaling current technology becomes a cost and fabrication challenge for $\mathrm{mmWave} / \mathrm{THz}$ multi-element arrays.

Alternatively, switching can be achieved with the use of tunable thin-film (2D or $2.5 \mathrm{D})$ materials; the electromagnetic properties of which (e.g. sheet resistance or permittivity/permeability) can be tuned over a large bandwidth with the use of an external mechanism (e.g. external field biasing or heating). Such materials include ferrite [31], liquid crystals [32], molybdenum disulfide $\left(\mathrm{MoS}_{2}\right)$ [33], black phosphorus $(\mathrm{BlPh})$ [34], vanadium dioxide $\left(\mathrm{VO}_{2}\right)$ [35]-[37], and graphene (GR) [38]-[49]. In the literature, $\mathrm{MoS}_{2}$ and $\mathrm{BlPh}$ switches are currently limited to lower frequencies $(<100 \mathrm{GHz})$ due to their high intrinsic impedances that demand for thin and long active device shapes to avoid excess losses; however, these configurations are highly affected by parasitics that become pronounced in high frequencies [33][34]. In addition, to the best of our knowledge, the fabrication of large-scale apertures with integrated switches demands for high-fabrication-yields, currently challenging with the use of these materials due to the growth and transfer processes [33][34]. Alternatively, $\mathrm{VO}_{2}$ switches have been proposed in $\mathrm{mmWave} / \mathrm{THz}$ bands with good performance (low insertion loss and high switching ratio) [35][36]; $\mathrm{VO}_{2}$ conductivity is thermally regulated and has been mostly exploited in power limiting switching topologies. Nevertheless, $\mathrm{VO}_{2}$ switching is sensitive to ambient temperature changes [37], a limiting factor for reliable compact multi-element designs that exhibit strong heat dissipation. In addition, the heaters of $\mathrm{VO}_{2}$ switches require at least $20-50 \mathrm{~mW}$ per element [35], thus power consumption is expected to skyrocket in multielement configurations with hundreds or even thousands of integrated switches. Although thin-film materials are probably in their infant in terms of usage in $\mathrm{mmWave} / \mathrm{THz}$ applications. Yet, there are indications that such tunable materials can provide key advantages over more traditional semiconductor technologies, including 1) wideband and higher cut-off frequency performance [37]-[43], 2) low-cost large-area fabrication [39][40], and 3) compatibility with standard substrate materials and nanofabrication tools [39].

In this study, we focus on graphene loaded (GL) coplanar transmission line (TL) switches, as illustrated in Fig. 1. Graphene is a 2D material that exhibits sheet resistance tunability over a great bandwidth (DC-1.5 THz) with the use of external biasing similarly to field-effect-transistors [38]-[49]. Additionally, the implementation of large-scale graphene reconfigurable apertures has been recently enabled with the development of a high-yield nanofabrication process that offers more than 92\% yield over a large area [39][40]. Even though graphene has a relatively low switching ratio $R_{\text {biased }}=300 \Omega / \square$ and $R_{\text {unbiased }}=1,500 \Omega / \square$ [41]-[44], these sheet resistances are considerably lower than $\mathrm{MoS}_{2}$ and $\mathrm{BlPh}$, leading to designs with fewer constraints regarding the channel/active device size, therefore fewer parasitic effects at $\mathrm{mmWave} / \mathrm{THz}$ bands. Several studies that propose graphene-loaded topologies include attenuators, switches, and reconfigurable power dividers [45]-[49]. These works include both experimental designs and/or theoretical studies for series or shunt configurations; however, an insight on the expected performance based on the configuration (series versus shunt topology) and the design parameters (e.g. characteristic impedance $-Z_{0}$ and graphene size) is missing. Specifically, the achievable ON/OFF ratio and insertion loss, when graphene is embedded in a switching topology, can differ from the sheet impedance ratio. For example, in [45], measurements up to 110 $\mathrm{GHz}$ of a shunt coplanar waveguide (CPW) graphene switch are presented, demonstrating the switching performance does not exceed $2 \mathrm{~dB}(I L \approx 10 d B)$. Conversely, in [47], a tunable graphene-based series microstrip attenuator is measured up to $20 \mathrm{GHz}$ achieving switching ratio of 3-5 $\mathrm{dB}(I L \approx 5 d B)$. Thus, which is the maximum switching performance for graphenebased switches and under what limitations?

The goal of this work is to methodically study GL TL switches and reveal the dependence of their performance on design parameters, including transmission line characteristic impedance, graphene patch geometry, scaling effects, and topology (series versus shunt). A partial study with respect to the aforementioned parameters is carried out in [46], where the performance of a series graphene switch is studied only for variable graphene patch widths. On the other hand, existing studies use equivalent models for graphene devices [47]-[49]. Namely, in [47] an equivalent model is used to extract the graphene sheet impedance values from the measured Sparameters of a microstrip attenuator. Moreover, in [48], an equivalent model approach is exploited to identify the losses in GL TLs, by carrying out full-wave simulations and then using the $A B C D$ parameters to extract the complex TL characteristics (complex-propagation constant and -characteristic impedance) for different frequencies. Finally, in [49] a one-port GL device is proposed and an equivalent model is used to extract the graphene sheet impedance values without investigating the effects of patch geometry on device performance. Nevertheless, no study examines in depth the switching performance with respect to the affecting parameters, especially for the various coplanar transmission line topologies presented in this work.

Herein, we model graphene using measured sheet impedance values ( $R_{\text {biased }}=300 \Omega / \square$ and $R_{\text {unbiased }}=1,500 \Omega / \square$ [41]-[44]) instead of theoretical models to include phenomena that affect the sheet impedance switching performance (e.g. lattice defects, grain size, etc.). As such, we identify the GL TL geometry that offers the best switching performance with limited graphene biased/unbiased ratio. Herein, we focus our effort on GL coplanar waveguides and striplines (CPW and CPS) switches, since these TLs are frequently used in $\mathrm{mmWave} / \mathrm{THz}$ integrated circuits (ICs) [50]-[53]. As such, we expand the theoretical models of the CPW and CPS TLs by incorporating the graphene sheet resistance. Furthermore, to identify the optimum switch topology using full-wave simulators based on multiple parameters is computationally intensive, especially for highfrequency circuits; therefore, the use of accurate equivalent models is a valuable tool for the design of multi-element configurations. Some prior results of this work are presented in [44]; however, that study is strictly limited to a brief discussion on general TL models (neither CPW nor CPS) and does not include the scaling effects and the parasitics of the devices. In 
this work, we extend the analysis including the dependence of the switching performance on device scaling, characteristic impedance, and graphene shape, incorporated in CPW and CPS TLs in the presence of parasitics. Moreover, our models incorporate graphene as a distributed sheet impedance in $\Omega / \square$; hence, they can be exploited for other loaded topologies that use materials characterized by sheet impedance (e.g. $\mathrm{VO}_{2}, \mathrm{MoS}_{2}$ etc.). Additionally, the proposed models can be used for rapid optimization processes or in conjunction with machine learning algorithms, where the generation of many design points is required. For example, machine learning algorithms are leveraged to optimize the antenna topology under certain constraints (e.g. gain, VSWR, return loss, etc.) as shown in [54]; these algorithms could now be extended for grapheneactuated reconfigurable antennas with embedded switches, which could be modelled as proposed herein.

The rest of the paper is organized as follows: In section II, we analyze the GL TLs using lumped models. Then, in section III, we investigate the performance of GL CPW switches based on various parameters and identify the optimum topology in terms of the switch geometrical features including device scaling, graphene squares, and transmission line characteristic impedance. Afterward, in section IV, we further the analysis of GL CPS switches. Finally, in section V, we discuss the estimated performance of the coplanar switch configurations for $2 \mathrm{D}$ materials with a wider tuning range of the impedance sheet.

\section{LUMPED-ELEMENT MODELING OF GRAPHENE SWITCHES}

In this section, we demonstrate the performance dependence of graphene-loaded transmission line switches based on graphene shape, characteristic impedance, and topology (series versus shunt). The equivalent circuits of the series and shunt graphene switches are depicted in Fig. 2a and their respective scattering parameters [55] are given by

$$
\begin{aligned}
& S_{11}^{\text {series }}=\frac{Z_{g r, \text { sheet }} / N}{2 Z_{o}+Z_{g r, \text { sheet }} / N}=S_{22}^{\text {series }} \\
& S_{21}^{\text {series }}=\frac{2 Z_{o}}{2 Z_{o}+Z_{g r, \text { sheet }} / N}=S_{12}^{\text {series }} \\
& S_{11}^{\text {shunt }}=\frac{-Z_{o}}{2 Z_{g r, \text { sheet }} / N+Z_{o}}=S_{22}^{\text {shunt }} \\
& S_{21}^{\text {shunt }}=\frac{2 Z_{g r, \text { sheet }} / N}{2 Z_{g r, \text { sheet }} / N+Z_{o}}=S_{12}^{\text {shunt }}
\end{aligned}
$$

where, $Z_{g r \text {,sheet }}$ is the sheet impedance of graphene in $\Omega / \square$ $\left(R_{\text {biased }}=300 \Omega / \square\right.$ and $R_{\text {unbiased }}=1,500 \Omega / \square[44]$ ) and $N$ is the number of graphene squares oriented in a line vertical to the current flow $\left(N_{\text {series }}=w / l\right.$ and $\left.N_{\text {shunt }}=l / w\right)$. Considering that graphene has a uniform current distribution due to small electrical size, the number of squares defines the total lumped impedance of the graphene; for example, if graphene is 5 squares, then $R_{\text {biased }}=60 \Omega$ and $R_{\text {unbiased }}=300 \Omega$. Moreover, in (1) and (2) the S-parameters depend only in the number of squares $N$ (ratio of $w$ over $l$ ); thus, we do not account for any scaling effects, which are thoroughly presented in the following sections.

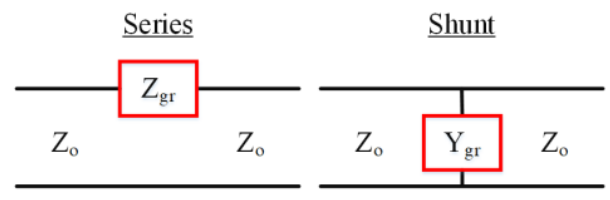

(a)

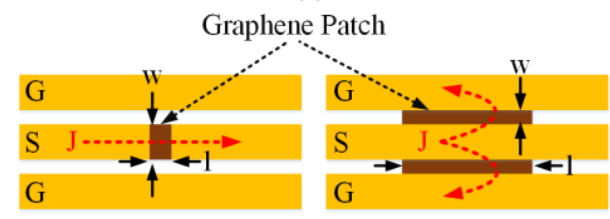

(b)

Fig. 2 (a) Graphene switches as lumped models (series and shunt) without parasitics and (b) the equivalent CPW topology paradigm (top view). $\underline{\text { Series }}$
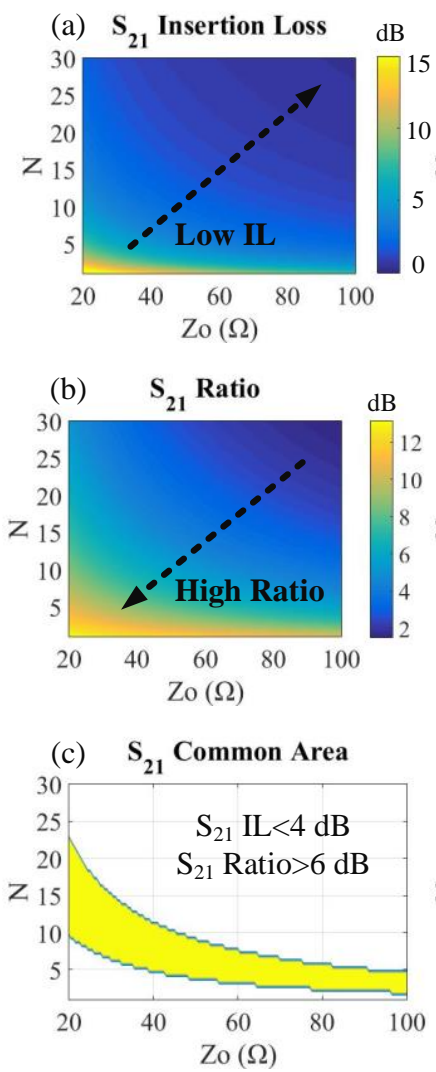

Shunt
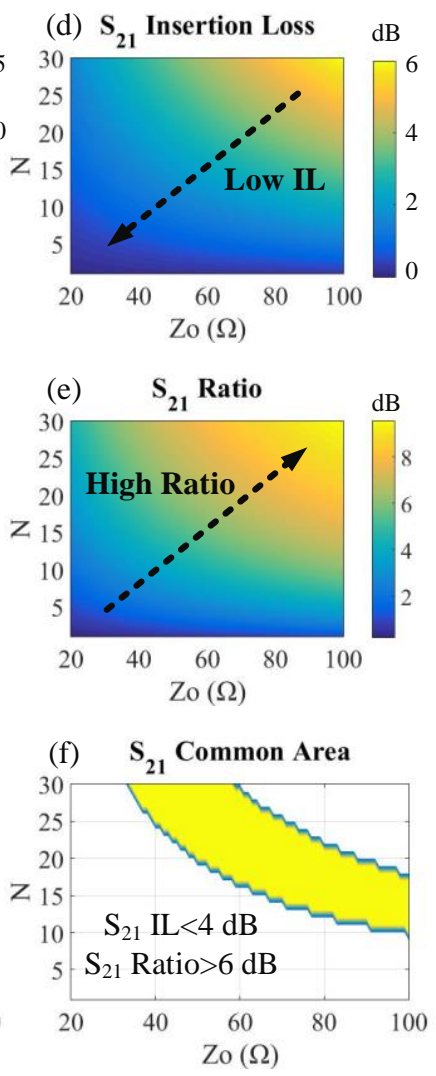

Fig. 3 The series/shunt switch topology (lumped): (a)/(d) Insertion loss, (b)/(e) ON/OFF ratio, and (c)/(f) the common area for given specifications.

The series (shunt) topology is in ON (OFF) state when graphene is biased and exhibits low impedance, thus leading to high (low) $S_{21}$. On the opposite, the series (shunt) switch is OFF $(\mathrm{ON})$ when graphene is unbiased-(high impedance) and the $S_{21}$ is low (high). The two switching performance metrics of interest are: i) insertion loss $\left(S_{21}\right)$ for the ON state and ii) the $S_{21}$ ratio between the $\mathrm{ON}$ and OFF states. Typically, improving the former reduces the latter and vice-versa. Therefore, switch design becomes a compromise between insertion loss and 


\section{ON/OFF ratio.}

The inverse proportionality between the switching ratio and the IL is also evident when simulating the topologies for variable $N$ and $Z_{0}$, as depicted in Figs. 3a-b,d-e. For specific switch operation (e.g. $I L<4 d B$ and $R>6 d B$ ), we can identify the range of $\left\{N, Z_{0}\right\}$ that satisfies these conditions (see Figs. 3c\&f).

We notice that both topologies can exhibit similar performance for the same graphene sheet impedance ratio ( 5 in this case). For example, if $Z_{0}=60 \Omega$ and $I L=3 d B$, both configurations can exhibit a maximum ON/OFF ratio of $7 \mathrm{~dB}$ (for different squares). However, as shown in the latter sections, the comparison is not always accurate, since the lumped modelling does not include any scaling effects that limit the performance due to the presence of parasitics. The effects of these parasitics are pronounced in higher-frequencies, since they offer a better path for the currents that flow through the switch, thus mitigating the performance.

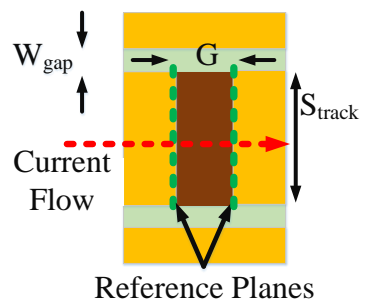

(a)

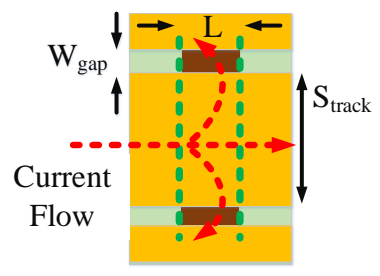

(c)

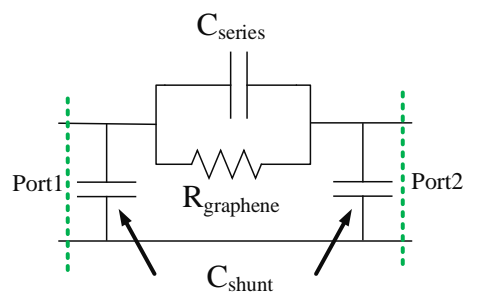

(b)

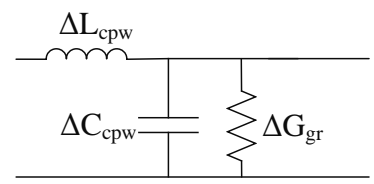

(d)
Fig. 4 The CPW (a) series and (c) shunt switch configurations (top-view) and in (b) and (d) the respective equivalent circuits.

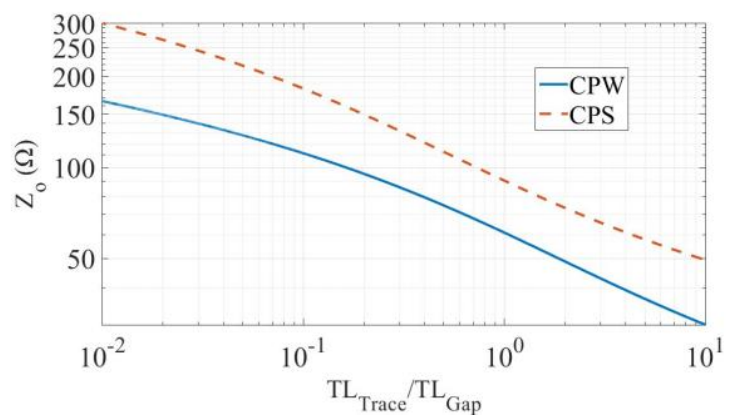

Fig. 5 The CPW and CPS characteristic impedance versus the TL trace over gap ratio [52].

\section{GRAPHENE LOADED CPW SWITCHES}

In this section, we expand the study of Section-II on the graphene switch performance, by including the scaling factor of the device for series and shunt topologies. The scaling factor affects the device parasitics (neglected in Section-II) and thus further investigation on the performance is needed with respect to the characteristic impedance, the number of graphene squares, and scaling factor. To carry out this study, we assume that the switches are implemented in a CPW topology, as depicted in Fig. 4.

\section{A. Series CPW Topology}

The series GL-CPW switch is modelled with an equivalent $\Pi$-network, as shown in Fig. 4b. The parasitic capacitances of the configuration depend on the geometrical features of the discontinuity [50]. Graphene sheet is modelled using a lumped resistor (Fig. 4b). The $A B C D$ parameters of the series GL-CPW switch are [55]

$$
A B C D=\left[\begin{array}{cc}
1+\frac{Y_{p}}{Y_{s}} & \frac{1}{Y_{p}} \\
2 Y_{p}+\frac{Y_{p}^{2}}{Y_{s}} & 1+\frac{Y_{p}}{Y_{s}}
\end{array}\right]
$$

where,

$$
Y_{s}=\frac{X_{s}+R_{g r}}{X_{s} R_{g r}}, \quad Y_{p}=\frac{1}{X_{p}}
$$

and,

$$
\begin{aligned}
& X_{s}=1 / i \omega C_{\text {series }}, \quad X_{p}=1 / i \omega C_{\text {shunt }}, \\
& \text { and } R_{g r}=Z_{\text {gr,sheet }} / N
\end{aligned}
$$

(a) $\mathrm{N}=1$ and $\mathrm{Zo}=80(\Omega)$

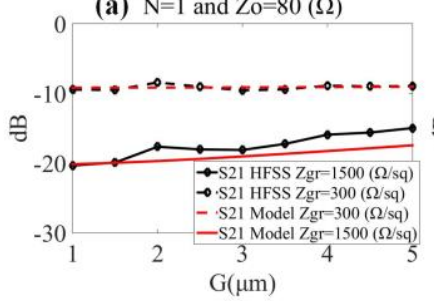

(c) $\mathrm{N}=6$ and $\mathrm{Zo}=50(\Omega)$

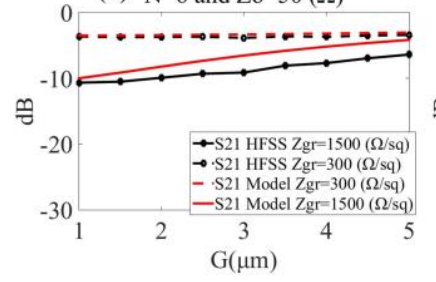

(b) $\mathrm{N}=1$ and $\mathrm{Zo}=80(\Omega)$

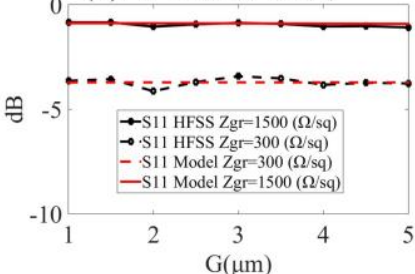

(d) $\mathrm{N}=6$ and $\mathrm{Zo}=50(\Omega)$

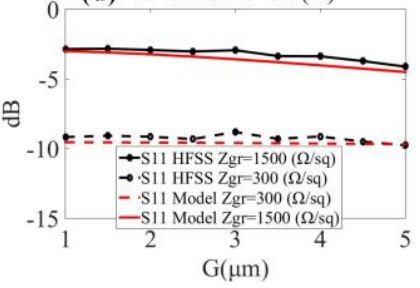

Fig. 6 The $S_{2 l}$ and $S_{I l}$ comparison between the proposed series GL-CPW switch model and full-wave simulations for various configurations.

To evaluate the accuracy of our model, we carry out fullwave simulations using a commercial finite element method (FEM) solver [56]. In these simulations, we fix the number of graphene squares $(N)$ and $C P W Z_{0}$ (we keep a fixed ratio of $S_{\text {track }} / W_{\text {gap }}$ as shown in Fig. 5), and vary the device length/scaling factor $(G)$. From the obtained results presented in Fig. 6, we observe that the model has good performance for various gap size $(G)$ values. The discrepancy observed in Fig. 6 c for high $G$ values is due to the model inaccuracies of the parasitic capacitance obtained from [50]. Namely, the effect of fringing fields is neglected in the models of [50], thus they become inaccurate for greater $G$ values. Moreover, if we followed the simplistic approach of Section II, the S-parameters (thus IL and ON/OFF ratio) of the devices would be constant 
regardless of the scaling factor $G$, since (1)-(2) depend only on $N$ and $Z_{0}$.

The simulation results are de-embedded using a thru-reflectline (TRL) calibration [57], eliminating any mismatch effects of the FEM solver's ports. As such, for each of the $G$ values, we simulated four configurations (3 TRL standards and 1 series GL-CPW switch). These simulations are carried out at $300 \mathrm{GHz}$ and the substrate is high resistivity silicon $\left(\varepsilon_{r}=11.9\right)$ (as for the rest of the paper), leading to dense meshes that increase the total simulation time. Namely, to obtain the S-matrix for each $G$-step of Fig. 6, approximately 3 minutes are needed (on a system with an Intel Xeon 6-core processor and 64 GB RAM). Therefore, if a design demands optimization based on $N, Z_{0}$, and $G$, with a fine step, the total full-wave simulation time becomes a bottleneck. Thus, the proposed models are an invaluable tool for the design of GL switches.

(a) Series Switch Algorithm

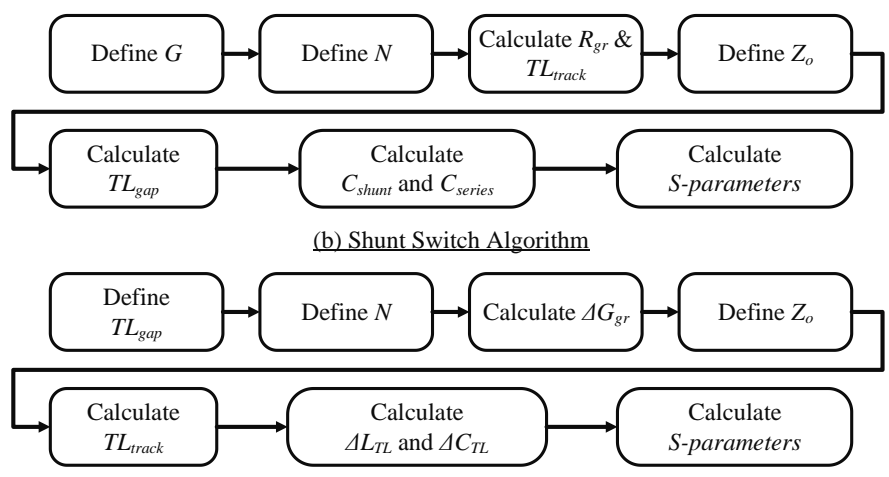

Fig. 7 The algorithms (a) series and (b) shunt, used to analyze the multidimensional problems based on the scaling factors, graphene squares, and $Z_{0}$.

The results of Fig. 6 are a small subset of the available topologies based on $Z_{0}, N$, and $G$ parameters. Hence, to identify the performance based on all the incorporated parameters; we need to carry out a scan for all the available combinations $\{G$, $\left.N, Z_{0}\right\}$. Explicitly, we use the algorithm given in Fig. 7a to calculate the S-parameters of the series GL-CPW switch. $Z_{0}$ (Fig. 5) is scanned between 20 and $120 \Omega\left(S_{\text {track }} / W_{\text {gap }}\right.$ ratio from 0.05 to 12 ), leading to low radiation losses and practically-sized features [52]. The obtained results are a 5-dimensional matrix (assuming the devices are reciprocal) that contains all the Sparameters for each available combination of $\left\{G, N, Z_{0}\right\}$, one for every switch state (ON/OFF). To observe the results, we first examine the IL and switching ratio of the series switch. For example, in Fig. 8a, the surfaces of constant IL $\left(S_{2 l} \mathrm{ON}\right)$ values are plotted; each point on these surfaces provides a combination of $\left\{G, N, Z_{0}\right\}$. Similarly, in Fig. 8b, surfaces of constant $S_{21}$ ON/OFF ratio values determine the respective combination of $\left\{G, N, Z_{0}\right\}$. We notice that increasing the number of graphene squares $N$, decreases the IL but also decreases the switching ratio. Similar trends are noticed for the other two parameters $G$ and $Z_{0}$, indicating that there is large design flexibility to achieve the desired specifications.
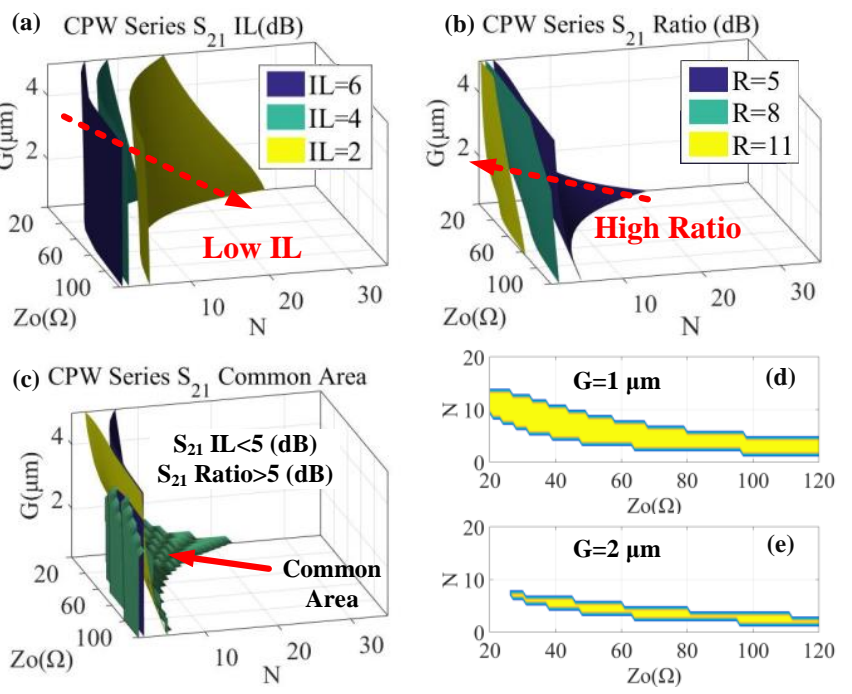

Fig. 8 The $S_{21}$ parameters of the series GL-CPW switch: (a) insertion loss, (b) ON/OFF ratio, (c) common locus (volumetric) for given criteria, and (d)-(e) the slices of the volumetric locus for different $G$ values.

In Fig. 8c, we depict the common area of $\left\{G, N, Z_{0}\right\}$ that follow the exemplary specifications of $I L<5 d B$ and switching ratio greater than $5 \mathrm{~dB}$. These results suggest that the series GLCPW switch depends on the gap size/scaling factor $(G)$. Namely, in Fig. 8d-e, we depict slices, for different $G$ values, of the common area from Fig. 8c. As expected, scaling the device size to smaller gap values decreases the parasitic effects, therefore, increasing the common area.

Examining the multi-dimensional data can be challenging to the designer and prioritizing specific parameters can help arrive faster in the desired switching topology. For example, by defining a desired/acceptable IL we can obtain the parameter space that satisfies the performance goal. As such, in Fig. 9 we present the ON/OFF ratio with respect to $\left\{G, N, Z_{0}\right\}$ for fixed IL values, observing a tradeoff between the IL and ON/OFF ratio. These maps are very useful since the designer can identify the desired ON/OFF ratio for given IL and obtain the geometrical characteristics of the design. Additionally, using the proposed models we obtain more than 600,000 S-matrices for all the $\left\{G, N, Z_{0}\right\}$ combinations, all these are calculated in less than 15 minutes using the proposed equivalent model. However, the same time using full-wave simulations would result in several months of run time.

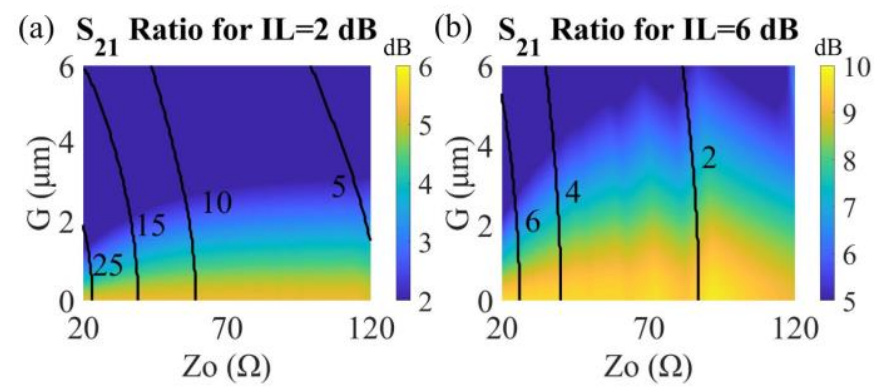

Fig. 9 The series GL-CPW switch 2D maps of ON/OFF ratio for fixed IL. With black contour, the number of squares is noted. 
(a) $\mathrm{N}=15$ and $\mathrm{Zo}_{\mathrm{o}}=90(\Omega$

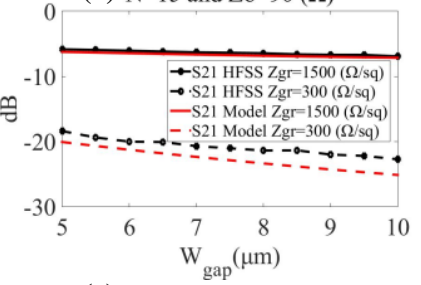

(c) $\mathrm{N}=30$ and $\mathrm{Zo}=40(\Omega)$

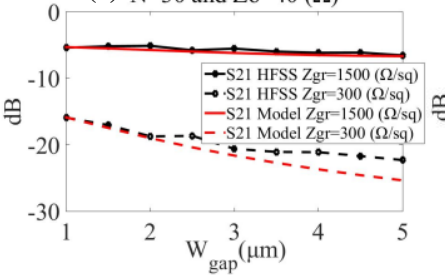

(b) $\mathrm{N}=15$ and $\mathrm{Zo}_{0}=90(\Omega)$

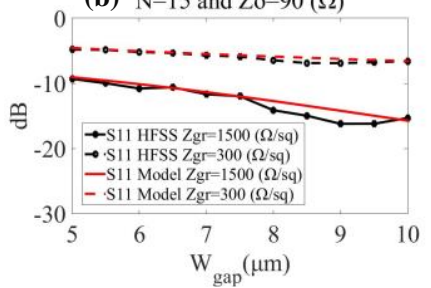

(d) $\mathrm{N}=30$ and $\mathrm{Zo}_{0}=40(\Omega)$

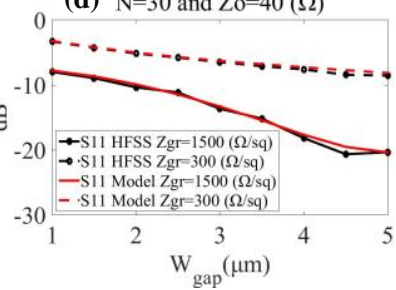

Fig. 10 The $S_{21}$ and $S_{11}$ comparison between the proposed shunt GL-CPW switch model and full-wave simulations for various configurations.

In the following sub-section, the case study of the shunt GLCPW switches is presented and a comparison between the series and the shunt topology is carried out in terms of switching performance.

\section{B. Shunt CPW Topology}

In this section, we present the model for the shunt GL-CPW switch shown in Fig. 4c. In this design, we incorporate graphene in the gaps of the CPW line and model the switch as a distributed transmission line. Graphene is modelled as a distributed admittance of $\Delta G_{g r}=2 N L / Z_{g r \text {, sheet }}$. The propagation constant and $Z_{0}$ of the GL-CPW are given by [48]

$$
\begin{aligned}
& \gamma_{g r}=\sqrt{(j \omega \Delta L)\left(\Delta G_{g r}+j \omega \Delta C\right)} \\
& Z_{o, g r}=\sqrt{(j \omega \Delta L) /\left(\Delta G_{g r}+j \omega \Delta C\right)}
\end{aligned}
$$

where $\triangle L$ and $\triangle C$ are the distributed CPW components obtained by [52]. Thus, the $A B C D$ parameters of the GL-CPW switch are [55]

$$
A B C D=\left[\begin{array}{cc}
\cosh (\gamma L) & Z_{o, g r} \sinh (\gamma L) \\
\frac{\sinh (\gamma L)}{Z_{o, g r}} & \cosh (\gamma L)
\end{array}\right]
$$

To validate the accuracy of the proposed model, we compare the analytical model results with full-wave simulations, as shown in Fig. 10. Similarly, to the series case, we keep $N$ and $Z_{0}$ fixed and vary $W_{\text {gap }}$ (scaling factor) of the CPW, observing a good agreement between our model and full-wave simulations.

Following the algorithm given in Fig. $7 \mathrm{~b}$, the S-matrices for all the available $\left\{W_{\text {gap }}, N, Z_{0}\right\}$ combinations are obtained, and in Fig. 11 the 3D isosurfaces of the $S_{21}$ are presented. Conversely, to the series case, the IL has an opposite trend with respect to $N$. The common region of the $S_{21}$ is given in Fig. 11c, and is significantly larger for the shunt GL-CPW switch compared to the series topology.

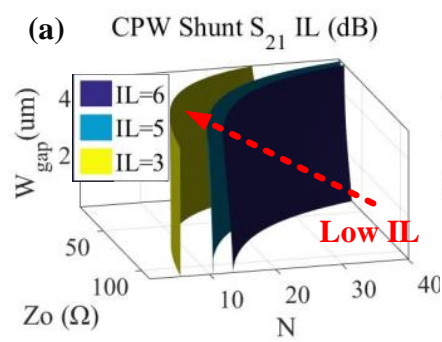

(b) CPW Shunt $\mathrm{S}_{21}$ Ratio (dB)

(c) CPW Shunt $\mathrm{S}_{21}$ Common Area
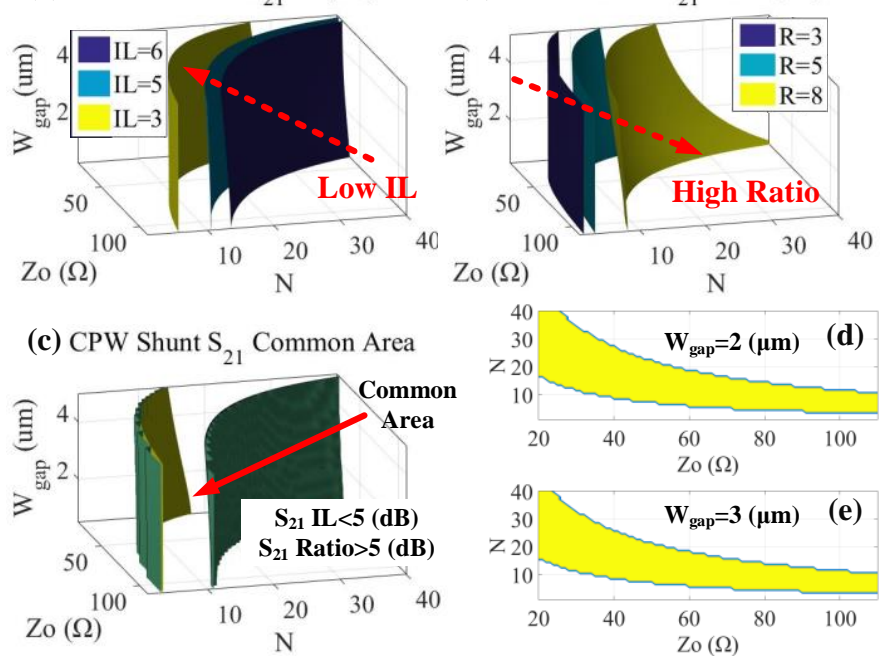

Fig. 11 The $S_{21}$ parameters of the shunt GL-CPW switch: (a) insertion loss, (b) ON/OFF ratio, (c) common locus (volumetric) for given criteria, and (d)-(e) the slices of the common for different $W_{\text {gap }}$ values.

In Fig. 11d-e the slices of the common areas (Fig. 11c) for different $W_{\text {gap }}$ values are given. Contrary to the series case (Fig. $8 \mathrm{~d}-\mathrm{e})$, the common area is not significantly limited by parasitics (series and shunt capacitances of Fig. 4b), hence offering greater flexibility for the design of GL switches. In addition, in Fig. 12 the $S_{21}$ ON/OFF ratio for fixed IL values is given with respect to $\left\{W_{g a p}, N, Z_{0}\right\}$. The results indicate that the switching performance improves with the larger scale of the devices, conversely to the series topology. Overall, the shunt topology offers better performance when considering fabrication limitations. For example, for $I L=6 d B$, the maximum achieved ratio is $19 \mathrm{~dB}$ for the shunt case, while for the series is less than $10 \mathrm{~dB}$. Hence, using our model we establish that the shunt switch design is preferable over the series in terms of IL and ON/OFF ratio.
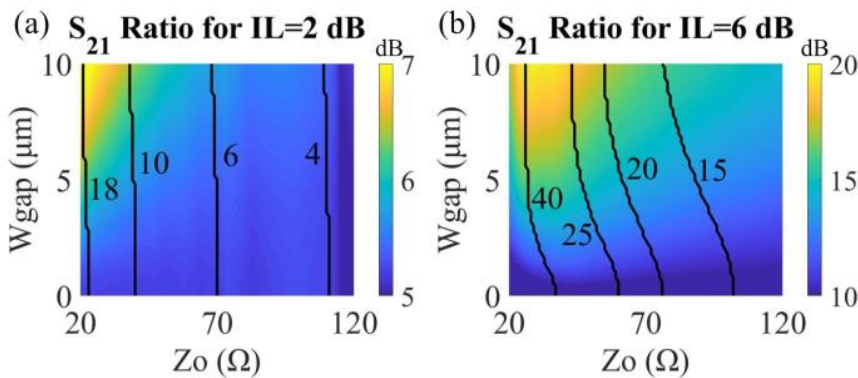

Fig. 12 The shunt GL-CPW switch 2D maps of ON/OFF ratio for fixed IL. With black contour, the number of squares is noted.

All the results of this section are obtained using the proposed equivalent model and suggest that the shunt topology outperforms the series. Nevertheless, we mention the following regarding the proposed designs: 1) The series case has better performance for smaller gap $(G)$ sizes $(<1 \mu \mathrm{m})$, which requires advanced/costly nanolithography processes (e.g. electron beam lithography-EBL) for fabrication. On the other hand, the performance of the shunt increases proportionally to the scaling factor, thus alleviating the cost of complex fabrication processes. However, as the CPW TL $W_{\text {gap }}$ size increases, radiation and higher-order mode losses increase. As shown in Fig. 10, the proposed model has good agreement with the full- 
wave simulations that include all these losses, but for electrically larger designs, radiation losses and higher-order modes (not accounted for herein) are expected to limit the switching performance.

\section{GRAPHENE LOADED CPS SWITCHES}

Another type of TL that are used in planar ICs is coplanar stripline, especially for cases where a higher $Z_{0}$ is preferred (see Fig. 5). In this section, we expand the theoretical modelling proposed in Section III, for the case of series/shunt GL-CPS switches. Similarly, to the CPW topology, we characterize the switching performance based on the scaling factor, characteristic impedance, and graphene shape (squares).

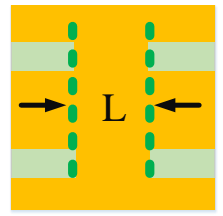

(a)

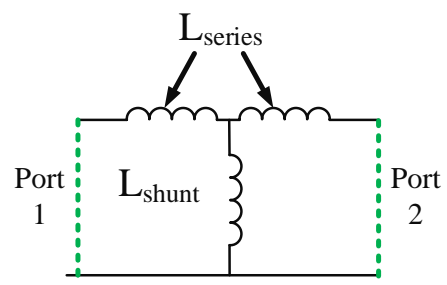

(c)

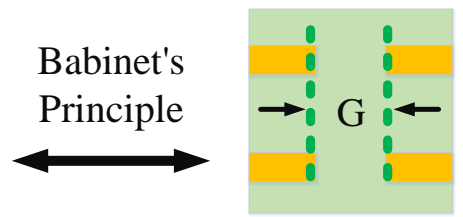

(b)

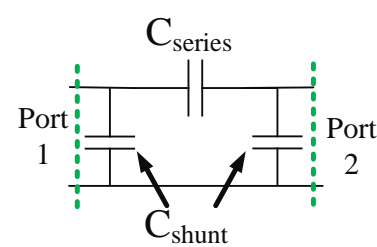

(d)
Fig. 13 (a) The CPW short discontinuity and (c) the equivalent circuit. Contrary, using Babinet's principle (b) the CPS gap discontinuity and (d) the equivalent circuit.

\section{A. Series CPS Topology}

To formulate the series GL-CPS switch, we first need to derive the equivalent circuit in the absence of graphene (parasitics), namely the series gap discontinuity. Unlike the CPW gap discontinuity, the CPS gap discontinuity's equivalent circuit models are not available in the existing literature in closed-form. Nevertheless, we can extract the equivalent models using Babinet's principle. The equivalent circuit of a shorted CPW is a T-network comprised of three inductors [50], as depicted in Fig. 13a\&c. This topology is complementary to the CPS gap discontinuity (Fig. 13 b\&d); hence using Babinet's principle the equivalent circuit of the CPS gap discontinuity is modelled with a П-network comprised of three capacitors. The expressions of the parasitic capacitances are obtained by [58]

$$
\begin{aligned}
& C_{\text {shunt }}=\frac{\varepsilon_{\text {eff }}}{(120 \pi)^{2}} L_{\text {series }} \\
& C_{\text {series }}=\frac{\varepsilon_{\text {eff }}}{(120 \pi)^{2}} L_{\text {shunt }}
\end{aligned}
$$

where, $\varepsilon_{e f f}=\left(\varepsilon_{r}+1\right) / 2, \varepsilon_{r}$ is the dielectric constant of the substrate, and $L_{\text {shunt }} / L_{\text {series }}$ are the inductances of the CPW short discontinuity given in [50], in closed form. The graphene patches placed in the gaps of the series GL-CPS switch are modelled with a resistor ( $\left.R_{\text {graphene }}\right)$, as shown in Fig. 14 . The proposed model shows very good agreement when compared with full-wave simulations for various switch parameters, as depicted in Fig. 15.

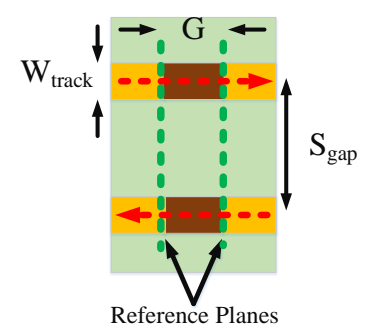

(a)

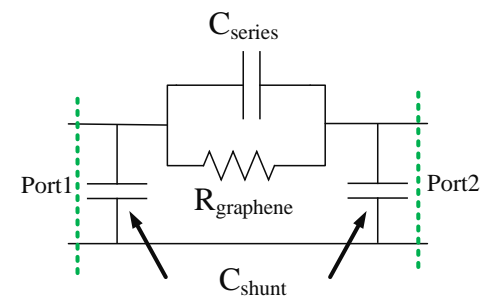

(b)
Fig. 14 (a) The series GL-CPS switch configuration (top-view) and (b) the equivalent circuit. (a) $\mathrm{N}=5$ and $\mathrm{Zo}=60(\Omega)$

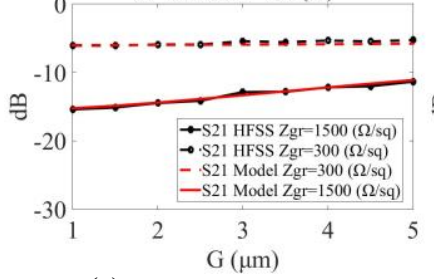

(c) $\mathrm{N}=10$ and $\mathrm{Zo}=110(\Omega)$

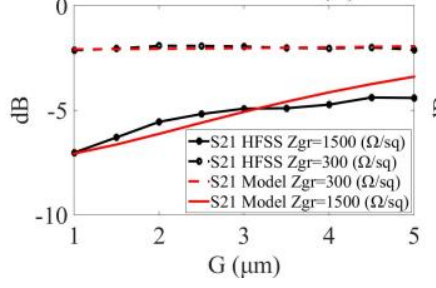

(b) $\mathrm{N}=5$ and $\mathrm{Zo}=60(\Omega)$

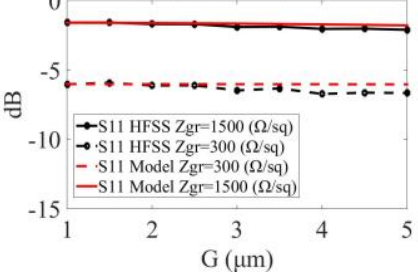

(d) $\mathrm{N}=10$ and $\mathrm{Zo}_{0}=110(\Omega)$

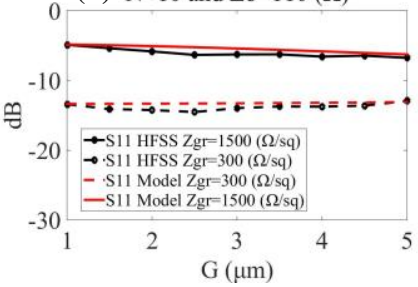

Fig. 15 The $S_{21}$ and $S_{11}$ comparison between the proposed series GL-CPS switch model and full-wave simulations for various configurations.

The switching performance with respect to the various parameters $\left\{G, N, Z_{0}\right\}$ follows similar trends to the results presented for the series GL-CPW switch (see Fig. 8), thus are not included in the manuscript.

In Fig. 16, the $S_{2 l}$ ON/OFF ratios are presented for two values of insertion loss. When compared to the same switch parameters of the series CPW topology (see Fig. 9), the series CPS $S_{21}$ ratio falls off slower for larger $G$ values and can have a potential advantage when gap minimum-dimensions are limited.
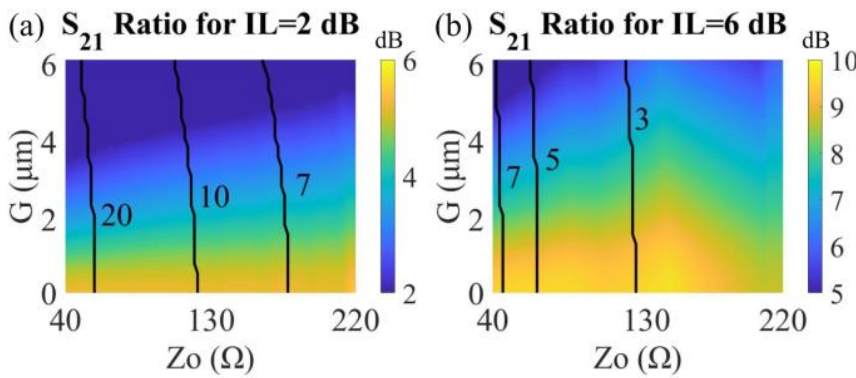

Fig. 16 The series GL-CPS switch 2D maps of ON/OFF ratio for fixed IL. With black contour, the number of squares is noted. 


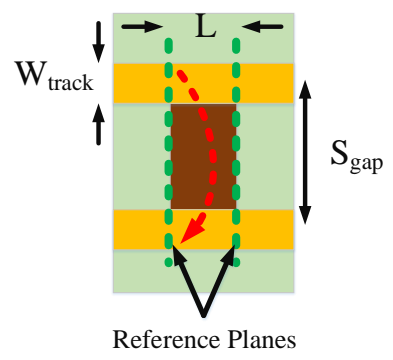

(a)

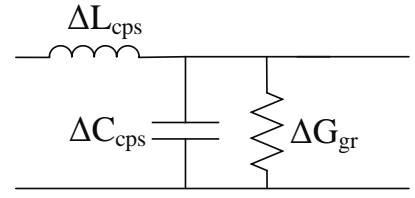

(b)

Fig. 17 (a) The shunt GL-CPS switch configuration (top-view) and (b) the equivalent circuit.

\section{B. Shunt CPS Topology}

For the shunt GL-CPS switch topology, we consider that graphene is placed in the gap between the signal and the ground traces, as is illustrated in Fig. 17a. The expressions for the distributed components are given in (6). In this case, we consider that the graphene distributed admittance is given by $\Delta G_{g r}=N L / Z_{g r \text {,sheet }}$. The $A B C D$ parameters are obtained using (7). The comparison between the full-wave simulations and the proposed model are depicted in Fig. 18, where a very good agreement is observed.

Similarly, to the series case, the switching performance trends with respect to the configuration parameters $\left\{W_{\text {gap }}, N\right.$, $Z_{0}$, are similar to the shunt CPW switch (see Fig. 11), thus are not included in the manuscript. Finally, in Fig. 19 the $S_{21}$ ON/OFF ratios are given for various ILs. From these graphs, we observe that the shunt CPS switch has very similar performance with the respective CPW switch.

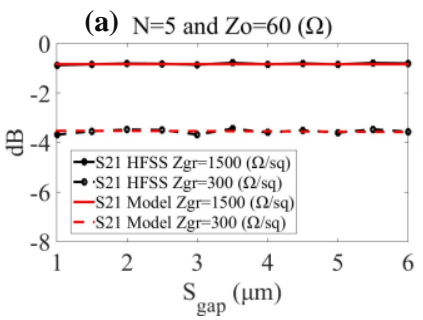

(c) $\mathrm{N}=25$ and $\mathrm{Zo}=110(\Omega)$

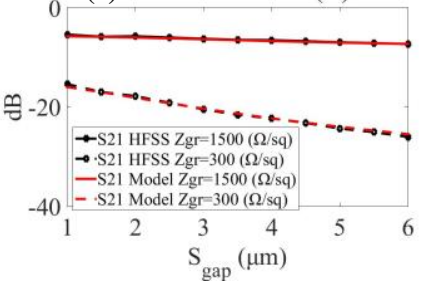

Fig. 18 The $S_{21}$ and $S_{11}$ comparison between the proposed shunt GL-CPS switch model and full-wave simulations for various configurations.

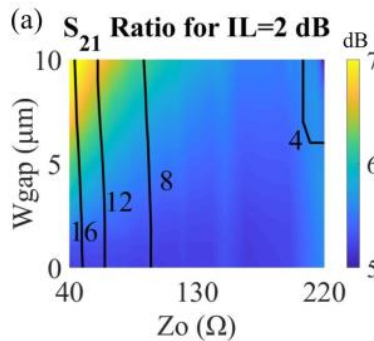

(b) $\mathrm{S}_{21}$ Ratio for $\mathrm{IL}=\mathbf{6} \mathrm{dB}$

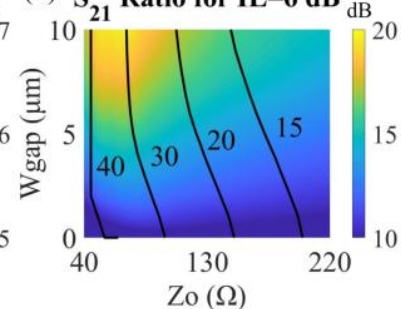

Fig. 19 The shunt GL-CPS switch 2D maps of ON/OFF ratio for fixed IL. With black contour, the number of squares is noted.

\section{DISCUSSION}

The theoretical models proposed in this work are used to reveal the performance of GL switches based on coplanar waveguide topologies. The presented results reveal the trends between the different series/shunt topologies with respect to geometrical parameters. From the obtained results, we conclude that the shunt switches are dominant over the series in terms of switching performance (IL and ON/OFF ratio). However, this model can be easily adjusted to other materials and/or graphene ratios. Specifically, graphene is expected to have a sheet impedance biased/unbiased ratio between 5-10 [41]-[49]. In this work, we chose 5 based on our previous experiments in the 220-330 GHz band [44]. However, if the fabrication process is optimized, the impedance ratio will increase. In addition, instead of monolayer graphene, the thin film could be comprised of graphene flakes that exhibit a higher ratio [47]. For example, if we consider a ratio of $10\left(R_{\text {biased }}=150 \Omega / \square\right.$ and $R_{\text {unbiased }}=1500 \Omega / \square$ ), the switching performance increases significantly both for the series and the shunt case, as shown in Fig. 20 (CPW topology). Namely, for the series GL-CPW switch, if the impedance ratio is 5 , and we consider an $I L=2 d B$, $Z_{0}=50 \Omega$, and $G=5 \mu \mathrm{m}$, the maximum switching ON/OFF ratio is $6 \mathrm{~dB}$, while for an impedance ratio of 10 , the respective ON/OFF switching ratio is $12 \mathrm{~dB}$. To compare with existing configurations, at $275 \mathrm{GHz}, \mathrm{VO}_{2}$ switches can achieve an ON/OFF ratio of $9 \mathrm{~dB}$ for an insertion loss of $2 \mathrm{~dB}$ [36].

Furthermore, in this work, the used graphene impedance values include the parasitic effects from the biasing structures (gates). Specifically, in our case study, we assume that graphene is biased with ion-gel gates, a polymer with losses of approximately less than $0.4 \mathrm{~dB} / 100 \mu \mathrm{m}$ at $300 \mathrm{GHz}$ [41]-[44]. Hence, our models, account for the total performance of the graphene switches, since the parasitic gating losses are taken into consideration. One limitation of this gating approach is the switching speed that we do not analyze herein since it exceeds the scope of this work. However, according to the existing literature, the switching speed of ion-gel gated graphene can reach a few $\mathrm{MHz}$ [42][43]. Finally, this approach can also be used for the design of reflection-based switches. In such topologies, the performance metrics are the return loss $(R L)$ and ON/OFF ratio of reflection coefficients $\left(S_{11}\right)$, which are expected to have opposite trends to the results presented herein, though similar performance in terms of $R L$ and ON/OFF ratio.
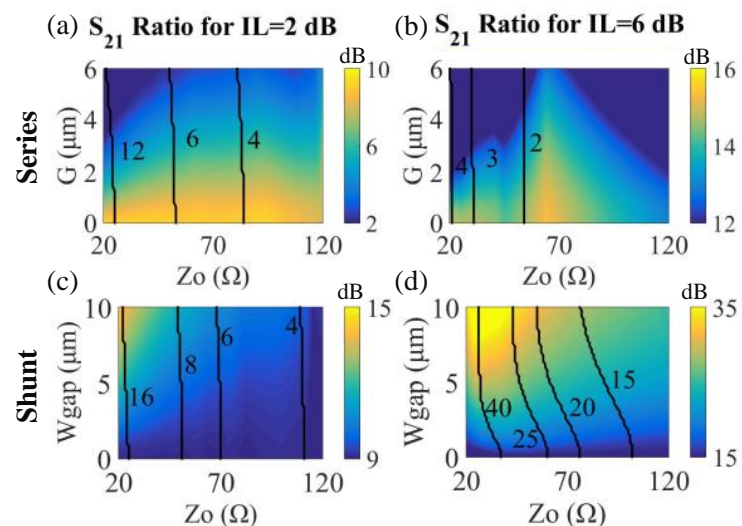

Fig. 20 The series and shunt GL-CPW switch 2D maps of ON/OFF ratio for fixed IL. These results are obtained for an impedance ratio of 10, instead of 5 as rest of the paper. The black contour indicates the number of squares $(\mathrm{N})$. 


\section{CONCLUSION}

Herein, we presented an in-depth analysis of graphene loaded switches for coplanar waveguide topologies (CPW and CPS). The study is carried out using equivalent models both for the series and shunt configurations with respect to various parameters including scaling, characteristic impedance, and graphene size. We validate the results of the proposed equivalent models using a FEM solver achieving good agreement between model and full-wave simulations. Afterward, we use the proposed models to obtain the S-matrices and identify the trends between the IL and ON/OFF ratios both for series and shunt GL- CPW and CPS switches. Alongside the theoretical models of the switches, the series CPS discontinuity equivalent circuit is presented for the first time using Babinet's principle. From the acquired data, we concluded that the shunt topology outmatches the series in terms of switching performance (both IL and ON/OFF ratio), both for the CPW and CPS topologies. This study would be impossible without the use of the proposed equivalent models, since, we calculated more than 2,400,000 S-matrices for the CPW and CPS cases, a herculean task using 3D full-wave solvers. The proposed methodology can be extended for other types of transmission lines (e.g. slotlines). Furthermore, other thin-film materials that are modelled by sheet impedance/conductance (e.g. $\mathrm{VO}_{2}$, $\mathrm{MoS}_{2}$, BIPh etc.) can be analyzed with the proposed approach.

\section{ACKNOWLEDGEMENTS}

This work is partly supported by the National Science Foundation under Grant CAREER ECCS-1847138.

\section{REFERENCES}

[1] T. S. Rappaport et al., "Wireless Communications and Applications Above $100 \mathrm{GHz}$ : Opportunities and Challenges for 6G and Beyond," IEEE Access, p. 1, 2019.

[2] P. C. Theofanopoulos, M. Sakr, and G. C. Trichopoulos, "Multistatic Terahertz Imaging Using the Radon Transform," IEEE Trans. Antennas Propag., p. 1, 2019.

[3] G. C. Trichopoulos, H. L. Mosbacker, D. Burdette, and K. Sertel, "A Broadband Focal Plane Array Camera for Real-time THz Imaging Applications," IEEE Trans. Antennas Propag., vol. 61, no. 4, pp. 17331740, 2013.

[4] O. Yurduseven, K. Cooper, and G. Chattopadhyay, "Point-SpreadFunction (PSF) Characterization of a 340-GHz Imaging Radar Using Acoustic Levitation," IEEE Trans. Terahertz Sci. Technol., vol. 9, no. 1, pp. 20-26, 2019.

[5] P. C. Theofanopoulos and G. C. Trichopoulos, "A Terahertz Microscopy Technique for Sweat Duct Detection," in 2018 IEEE/MTT-S International Microwave Symposium - IMS, 2018, pp. 864-867.

[6] K. Liu et al., "100 Gbit/s THz Photonic Wireless Transmission in the 350$\mathrm{GHz}$ Band With Extended Reach," IEEE Photonics Technol. Lett., vol. 30, no. 11, pp. 1064-1067, 2018.

[7] X. Li et al., " $120 \mathrm{~Gb} / \mathrm{s}$ Wireless Terahertz-Wave Signal Delivery by 375 GHz-500 GHz Multi-Carrier in a $2 \times 2$ MIMO System," J. Light. Technol., vol. 37, no. 2, pp. 606-611, 2019.

[8] V. K. Chinni et al., "Single-channel $100 \mathrm{Gbit} / \mathrm{s}$ transmission using III-V UTC-PDs for future IEEE $802.15 .3 \mathrm{~d}$ wireless links in the $300 \mathrm{GHz}$ band," Electron. Lett., vol. 54, no. 10, pp. 638-640, 2018.

[9] S. Zhang, C. Guo, T. Wang, and W. Zhang, "ON-OFF Analog Beamforming for Massive MIMO," IEEE Trans. Veh. Technol., vol. 67, no. 5, pp. 4113-4123, 2018.

[10] X. G. Zhang, W. X. Jiang, H. W. Tian, Z. X. Wang, Q. Wang and T. J. Cui, "Pattern-Reconfigurable Planar Array Antenna Characterized by Digital Coding Method," in IEEE Transactions on Antennas and Propagation.
[11] H. Kamoda, T. Iwasaki, J. Tsumochi, T. Kuki, and O. Hashimoto, "60GHz Electronically Reconfigurable Large Reflectarray Using Single-Bit Phase Shifters," IEEE Trans. Antennas Propag., vol. 59, no. 7, pp. 25242531,2011

[12] E. Carrasco, M. Barba, and J. A. Encinar, "X-Band Reflectarray Antenna With Switching-Beam Using PIN Diodes and Gathered Elements," IEEE Trans. Antennas Propag., vol. 60, no. 12, pp. 5700-5708, 2012.

[13] H. Yang et al., "A programmable metasurface with dynamic polarization, scattering and focusing control," Sci. Rep., vol. 6, p. 35692, Oct. 2016.

[14] C. Huang, C. Zhang, J. Yang, B. Sun, B. Zhao, and X. Luo, "Reconfigurable Metasurface for Multifunctional Control of Electromagnetic Waves," Adv. Opt. Mater., vol. 5, no. 22, p. 1700485 , Nov. 2017.

[15] S. Costanzo, F. Venneri, A. Raffo, and G. Di Massa, "Dual-Layer SingleVaractor Driven Reflectarray Cell for Broad-Band Beam-Steering and Frequency Tunable Applications," IEEE Access, vol. 6, pp. 71793$71800,2018$.

[16] Y. Han, W. Tang, S. Jin, C. Wen and X. Ma, "Large Intelligent SurfaceAssisted Wireless Communication Exploiting Statistical CSI," in IEEE Transactions on Vehicular Technology, vol. 68, no. 8, pp. 8238-8242, Aug. 2019.

[17] H. Yang et al., "A Study of Phase Quantization Effects for Reconfigurable Reflectarray Antennas," IEEE Antennas Wirel. Propag. Lett., vol. 16, pp. 302-305, 2017.

[18] K. Sengupta and A. Hajimiri, "A 0.28 THz Power-Generation and BeamSteering Array in CMOS Based on Distributed Active Radiators," IEEE J. Solid-State Circuits, vol. 47, no. 12, pp. 3013-3031, 2012.

[19] A. C.. Ulusoy et al., "A Low-Loss and High Isolation D-Band SPDT Switch Utilizing Deep-Saturated SiGe HBTs," IEEE Microw. Wirel. Components Lett., vol. 24, no. 6, pp. 400-402, 2014.

[20] R. L. Schmid, A. Ç. Ulusoy, P. Song, and J. D. Cressler, "A 94 GHz, 1.4 dB Insertion Loss Single-Pole Double-Throw Switch Using ReverseSaturated SiGe HBTs," IEEE Microw. Wirel. Components Lett., vol. 24, no. 1, pp. 56-58, 2014.

[21] Y. Yang, O. D. Gurbuz, and G. M. Rebeiz, "An Eight-Element 370-410GHz Phased-Array Transmitter in 45-nm CMOS SOI With Peak EIRP of 8-8.5 dBm," IEEE Trans. Microw. Theory Tech., vol. 64, no. 12, pp. $4241-4249,2016$.

[22] M. Schröter et al., "SiGe HBT Technology: Future Trends and TCADBased Roadmap," Proc. IEEE, vol. 105, no. 6, pp. 1068-1086, 2017.

[23] Z. Iskandar, A. Siligaris, J. Lugo-Alvarez, E. Pistono, and P. Ferrari, “A 270-to-300 GHz LO-Path Phase Shifting Architecture for Sub-mm-wave Phased Arrays," in 2018 48th European Microwave Conference (EuMC), 2018, pp. 804-806.

[24] S. Lim, H. Koo, C. Kim, J. Kim, J. Rieh, and S. Hong, "290-GHz 17-dB ON-/OFF-Ratio Modulator With Resonance Control Varactors," IEEE Microw. Wirel. Components Lett., vol. 29, no. 1, pp. 50-52, 2019.

[25] F. Thome, M. Ohlrogge, A. Leuther, M. Schlechtweg, and O. Ambacher, "An investigation of millimeter wave switches based on shunt transistors including SPDT SWITCH MMICs up to $300 \mathrm{GHz}$," in 2016 IEEE MTTS International Microwave Symposium (IMS), 2016, pp. 1-4.

[26] D. Müller et al., "A Novel Unit Cell for Active Switches in the MillimeterWave Frequency Range," J. Infrared, Millimeter, Terahertz Waves, vol. 39, no. 2, pp. 161-176, 2018.

[27] T. Shivan et al., "220-325 GHz high-isolation SPDT switch in InP DHBT technology," Electron. Lett., vol. 54, no. 21, pp. 1222-1224, 2018.

[28] F. Thome and O. Ambacher, "Highly Isolating and Broadband SinglePole Double-Throw Switches for Millimeter-Wave Applications Up to 330 GHz," IEEE Trans. Microw. Theory Tech., vol. 66, no. 4, pp. 19982009, 2018.

[29] http://europractice-ic.com/wpcontent/uploads/2019/06/190517_MPW2019-general-v7.0.pdf

[30] J. D. Cressler et al., "SiGe Technology as a Millimeter-Wave Platform: Scaling Issues, Reliability Physics, Circuit Performance, and New Opportunities," in 2016 IEEE Compound Semiconductor Integrated Circuit Symposium (CSICS), 2016, pp. 1-13.

[31] P. C. Theofanopoulos, C. S. Lavranos, K. E. Z0iros, G. C. Trichopoulos, G. Granet, and G. A. Kyriacou, "Investigation of Nonreciprocal Dispersion Phenomena in Anisotropic Periodic Structures Based on a Curvilinear FDFD Method," IEEE Trans. Microw. Theory Tech., vol. 65, no. 1, pp. 36-49, 2017.

[32] M. Jost, R. Reese, M. Nickel, H. Maune, and R. Jakoby, "Fully dielectric interference-based SPDT with liquid crystal phase shifters," IET Microwaves, Antennas Propag., vol. 12, no. 6, pp. 850-857, 2018. 
[33] M. Kim, S. Park, A. Sanne, S. K. Banerjee, and D. Akinwande, "Towards mm-wave nanoelectronics and RF switches using MoS22D Semiconductor," in 2018 IEEE/MTT-S International Microwave Symposium - IMS, 2018, pp. 352-354.

[34] H. Huang, B. Jiang, X. Zou, X. Zhao, and L. Liao, "Black phosphorus electronics," Sci. Bull., vol. 64, no. 15, pp. 1067-1079, 2019.

[35] C. Hillman, P. A. Stupar, and Z. Griffith, "VO2 Switches for Millimeter and Submillimeter-Wave Applications," in 2015 IEEE Compound Semiconductor Integrated Circuit Symposium (CSICS), 2015, pp. 1-4.

[36] C. Hillman, P. Stupar, and Z. Griffith, "Scaleable vanadium dioxide switches with submillimeterwave bandwidth: VO2 switches with impoved RF bandwidth and power handling," in 2017 IEEE Compound Semiconductor Integrated Circuit Symposium (CSICS), 2017, pp. 1-4.

[37] H. Madan et al., "26.5 Terahertz electrically triggered RF switch on epitaxial VO2-on-Sapphire (VOS) wafer," in 2015 IEEE International Electron Devices Meeting (IEDM), 2015, pp. 9.3.1-9.3.4.

[38] D. Fadil et al., "2D-Graphene Epitaxy on SiC for RF Application: Fabrication, Electrical Characterization and Noise Performance," in 2018 IEEE/MTT-S International Microwave Symposium - IMS, 2018, pp. 228231.

[39] P. C. Theofanopoulos, S. Ageno, Y. Guo, S. Kale, Q. H. Wang, and G. C. Trichopoulos, "High-yield fabrication method for high-frequency graphene devices using titanium sacrificial layers," J. Vac. Sci. Technol. $B$, vol. 37, no. 4, p. 41801, Jun. 2019.

[40] P. C. Theofanopoulos and G. C. Trichopoulos, "On-Wafer Graphene Devices for THz Applications Using a High-Yield Fabrication Process," 2019 IEEE MTT-S International Microwave Symposium (IMS), Boston, MA, USA, 2019, pp. 1107-1110.

[41] Z. Miao et al., "Widely Tunable Terahertz Phase Modulation with GateControlled Graphene Metasurfaces," Phys. Rev. X, vol. 5, no. 4, p. 41027, Nov. 2015.

[42] J. Liu et al., "Flexible terahertz modulator based on coplanar-gate graphene field-effect transistor structure," Opt. Lett., vol. 41, no. 4, pp. 816-819, Feb. 2016.

[43] N. Kakenov, M. S. Ergoktas, O. Balci, and C. Kocabas, "Graphene based terahertz phase modulators," 2D Mater., vol. 5, no. 3, p. 035018, May 2018.

[44] P. C. Theofanopoulos and G. C. Trichopoulos, "Toward Large-Scale Dynamically Reconfigurable Apertures Using Graphene," 2019 IEEE International Symposium on Antennas and Propagation and USNC-URSI Radio Science Meeting, Atlanta, GA, USA, 2019, pp. 511-512.

[45] M. Dragoman et al., "Coplanar waveguide on graphene in the range 40 MHz-110 GHz,” Appl. Phys. Lett., vol. 99, no. 3, p. 33112, Jul. 2011.

[46] H.-J. Lee, E. Kim, W.-J. Lee, and J. Jung, "RF transmission properties of graphene monolayers with width variation," Phys. status solidi - Rapid Res. Lett., vol. 6, no. 1, pp. 19-21, Jan. 2012.

[47] L. Pierantoni et al., "Broadband Microwave Attenuator Based on Few Layer Graphene Flakes," IEEE Trans. Microw. Theory Tech., vol. 63, no. 8, pp. 2491-2497, 2015.

[48] Y. Wu, M. Qu, and Y. Liu, "A Generalized Lossy Transmission-Line Model for Tunable Graphene-Based Transmission Lines with Attenuation Phenomenon," Sci. Rep., vol. 6, p. 31760, Aug. 2016.

[49] J. Judek, M. Zdrojek, J. Sobieski, A. Przewłoka, and J. K. Piotrowski, "Characterization of the CVD Graphene Monolayer as an Active Element of a One-Port Microwave Device," IEEE Trans. Electron Devices, vol. 64, no. 10, pp. 4340-4345, 2017.

[50] N. Dib, "Comprehensive study of CAD models of several coplanar waveguide (CPW) discontinuities," IEE Proc. - Microwaves, Antennas Propag., vol. 152, no. 2, pp. 69-76, 2005.

[51] L. Zheng and A. Weisshaar, "Parameterised equivalent circuit model of frequency-dependent resistance and inductance for on-chip coplanar waveguides up to $110 \mathrm{GHz}$," Electron. Lett., vol. 54, no. 16, pp. 986-988, 2018.

[52] Rainee N. Simons, Coplanar Waveguide Circuits, Components, and Systems, 1st ed. New York, NY, USA: Wiley, 2001.

[53] Y. J. Guo, K. Da Xu, and X. Tang, "Spoof plasmonic waveguide developed from coplanar stripline for strongly confined terahertz propagation and its application in microwave filters," Opt. Express, vol. 26, no. 8, pp. 10589-10598, 2018.

[54] B. Liu, H. Aliakbarian, Z. Ma, G. A. E. Vandenbosch, G. Gielen, and P. Excell, "An Efficient Method for Antenna Design Optimization Based on Evolutionary Computation and Machine Learning Techniques," IEEE Trans. Antennas Propag., vol. 62, no. 1, pp. 7-18, 2014.

[55] D. M. Pozar, Microwave Engineering, 2nd ed. New York, NY, USA: Wiley, 1998. [56] ANSYS Electronics Desktop available at:
https://www.ansys.com/products/electronics/ansys-electronics-desktop

[57] D. Rytting, "Network analyzer error models and calibration methods," White Paper, Sep. 1998.

[58] W. J. Getsinger, "Circuit Duals on Planar Transmission Media," in 1983 IEEE MTT-S International Microwave Symposium Digest, 1983, pp. 154156. 